\section{Vertical cavity lasers on p-doped substrates}

K.L. Lear, H.Q. Hou, J.J. Banas, B.E. Hammons, J. Furioli and M. Osiński

Indexing terms: Vertical cavity surface emitting lasers, Semiconductor junction lasers

Vertical cavity surface emitting laser (VCSEL) diodes fabricated with inverted polarity, i.e. $p$-type bottom mirror and $n$-type top mirror, are reported with lower resistance and diode voltage and comparable output characteristics relative to similar conventional, non-inverted structures.

Most laser diodes are fabricated on $n$-doped substrates with $n$ doped material below the active region and $p$-doped material above it. However, both circuit and device performance considerations for arrays of vertical cavity surface emitting lasers (VCSELs) motivate inverting this polarity so that the substrate and lower epitaxial layers are $p$-doped and the upper epitaxial layers are $n$ doped. From a circuit perspective, this latter configuration allows simple fabrication of common anode laser arrays that can be driven with open collector $n p n$ transistors which typically perform better than $p n p$ transistors.

While consideration of $n$-doped and $p$-doped mirror properties suggests that $p$-substrate VCSELs should perform better than their $n$-substrate counterparts [1], such experimental results have not been reported. Results from external cavity VCSELs based on $p$ substrates have been published [2] but are not readily compared with purely monolithic structures. A more recent report of similar VCSELs with both types of substrates indicated that the performance of the $p$-substrate devices lagged that of the $n$-substrate devices [3]. Here, we report state-of-the-art, oxide-confined, $p$-substrate VCSELs with characteristics equal to or better than comparable $n$-substrate devices.

Material issues including the stability of dopants and the quality of substrates may have limited previous efforts to realise $p$-substrate VCSELs. Some $p$-type dopants such as zinc and beryllium are more diffusive or prone to surface segregation [4] than silicon, the most common $n$-type dopant. These properties can result in the movement of significant dopant concentrations from underlying material into the active region, reducing optical efficiency. This problem is enhanced in thick VCSEL structures with high aluminum content that require long growths at high temperatures. In general, $p$-type and semi-insulating substrates also have higher concentrations of defects than $n$-type substrates. Previous attempts at Sandia to realise $p$-substrate VCSELs using molecular beam epitaxy with beryllium doping have been unsuccessful. The structures presented here were produced with metalorganic chemical vapour deposition using $\mathrm{CCl}_{4}$ as the source for carbon as a low diffusivity, more readily incorporated $p$-type dopant. A constant growth temperature of $750^{\circ} \mathrm{C}$ was used throughout the structures, except for the heavily doped contact layers. Decreasing the growth temperature in the active region could lead to enhanced carbon incorporation from any residual $\mathrm{CCl}_{4}$ and was thus avoided [5]. The substrates were produced by vertical gradient freeze and had doping concentrations of $3 \times 10^{18} / \mathrm{cm}^{3}$ of zinc or $2 \times 10^{18} / \mathrm{cm}^{3}$ of silicon. The etch pit density of the $p$-substrates was $5000 / \mathrm{cm}^{2}$.

Lasers made on two $n$-substrate and one $p$-substrate wafers were examined. All the samples had five GaAs quantum wells in a single wavelength cavity with the composition graded between $\mathrm{Al}_{0.5} \mathrm{Ga}_{0.5}$ As and $\mathrm{Al}_{0.2} \mathrm{Ga}_{0.8}$ As. The two $n$-substrate samples had 40 period, silicon doped lower mirrors and 20 period, carbon doped upper mirrors and were nominally identical except for being offset in thicknesses so that the cavity wavelengths were $\sim 850$ and $830 \mathrm{~nm}$. The $p$-substrate sample had a 40 period, carbon doped lower mirror and 19 period, silicon doped upper mirror and an approximate wavelength of $840 \mathrm{~nm}$. Within each mirror period, the minimum aluminum mole fraction was 0.16 and the maximum varied between 0.94 and 0.97 for all of the mirror periods, except for the $p$-type mirror period next to the cavity, which incorporated $\mathrm{Al}_{0.98} \mathrm{Ga}_{0.02} \mathrm{As}$ to selectively oxidise a confinement layer at this position [6]. Note that the confinement layer defines a current constriction which is just above the cavity for the $n$-substrate samples and just below the cavity for the $p$-substrate sample. In both cases, placement of the oxide in the $p$-mirror may be preferred because of its lower vertical resistance in the constriction as noted below. The graded regions of each mirror period were doped $3-4 \times 10^{18 /}$ $\mathrm{cm}^{3}$. Outside the graded regions, the doping levels varied from $1 \times$ $10^{18} / \mathrm{cm}^{3}$ near the cavity to $3 \times 10^{18} / \mathrm{cm}^{3}$ far from the cavity. Heavily doped layers of a half-wavelength of $\mathrm{Al}_{0.16} \mathrm{Ga}_{0.84} \mathrm{As}$ and $150 \AA$ thick GaAs cap completed all the samples. These layers are used to improve current spreading [7] and reduce contact resistance, respectively. Carbon doping concentrations of $3 \times 10^{1 \%} / \mathrm{cm}^{3}$ and silicon doping concentrations of $4 \times 10^{18} / \mathrm{cm}^{3}$ were used in these last layers. The order of magnitude higher doping concentration facilitates non-alloyed $p$-type ohmic contacts to the $n$-substrate sample, while alloyed $n$-type contacts are required for the $p$-substrate sample.

The primary benefit anticipated from $p$-substrate VCSELs is reduced series resistance from the increased carrier mobility and thus lateral conductivity of the upper $n$-type mirror. Because $n$ type mirror vertical conductivity (perpendicular to the mirror layers) is typically limited by interfacial rather than bulk resistivity, $n$ type mirrors have in fact shown nonlinear current voltage characteristics with higher voltage drops than well graded $p$-type mirrors at low to moderate current densities $[8,9]$. Hence, placing the $n$ mirror above the active region, where the direction of current flow is predominantly lateral, reduces the total resistance of VCSELs with annular contacts. Prior to evaluating devices, it is beneficial to quantify the difference in lateral conductivity of the upper mirror layers in the epitaxial structures, described in the preceeding paragraph. The sheet resistances calculated from a simplified laminar model, based on composition and doping dependent mobility and intended dopant, rather than carrier distributions are 110 and $14 \Omega / \square$ for the two $p$-mirrors (on the $n$-substrate samples) and the $n$-mirror (on the $p$-substrate sample), respectively. The sheet resistances measured using a four-point probe were 80 and $83 \Omega / \square$ for the two $p$-mirrors and $35 \Omega / \square]$ for the $n$-mirror. The variation from the calculated values can be attributed to uncertainties in actual doping levels and the redistribution of carriers. The measured value for the $n$-mirror may also be higher than anticipated due to the higher vertical resistance interfering with the four point probe measurement. Beyond the simple resistance of the device, the low lateral resistance of the $n$-mirror should also result in more uniform current injection which promotes singlemode behaviour [2].

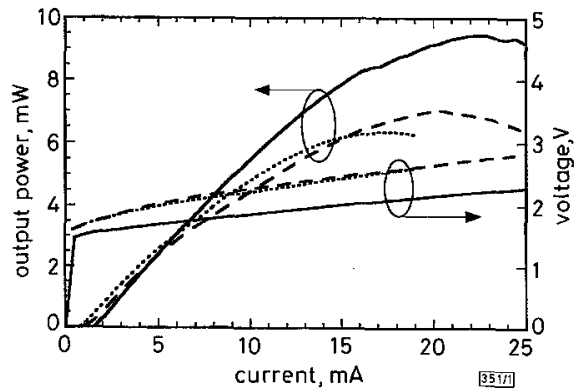

Fig. 1 Room temperature, $C W$ light and voltage against current characteristics for $9.1 \mu \mathrm{m}$ diameter, $840 \mathrm{~nm}$ p-substrate VCSEL, $9.1 \mu \mathrm{m}$ diameter, $830 \mathrm{~nm}$ n-substrate VCSEL, and $8.2 \mu \mathrm{m}$ diameter, $850 \mathrm{~nm} n$ substrate VCSEL

\section{$840 \mathrm{~nm}$ p-substrate VCSEL}

$830 \mathrm{~nm} n$-substrate VCSEL

$---850 \mathrm{~nm} n$-substrate VCSEL

The measured characteristics of $n$ - and $p$-substrate oxide-confined VCSELs are compared in Fig. 1. The slight differences in threshold current and slope efficiencies just above threshold are consistent with the higher output coupling of the 19 period $n$-mirror compared to the 20-period $p$-mirrors. Most notable, the $p$-substrate laser has a lower voltage drop. Its reduced heating due to the lower voltage drop also extends the maximum power point to a higher current of $23 \mathrm{~mA}$ before thermal rollover. Both the $n$-substrate lasers rollover at lower currents even though their wavelengths bracket the $p$-substrate laser's wavelength, indicating that the higher current operation of the $p$-substrate laser cannot be attributed to gain-cavity mode wavelength alignment.

To quantify the difference in the electrical characteristics of the $n$ - and $p$-substrate VCSELs, the size dependent resistance and extrapolated zero current voltage are shown in Figs. 2 and 3. The sizes of the oxide apertures, produced from round mesas were measured from the nearfield emission image at low currents. The 
resistance is calculated between the maximum efficiency and maximum power points where the current-voltage curve is most linear, and this resistance along with the voltage at either point is used to extrapolate the voltage to zero current. The $n$-substrate laser resistances are 1.3 to 1.8 times higher than the $p$-substrate laser resistances. A power law fit shows the resistance is proportional to the (diameter) $)^{-n}$, where $n=1.0$ for the $p$-substrate devices and $n \simeq$ 1.16 for the $n$-substrate devices. This exponent indicates that in both cases the resistance is dominated by contact, lateral, and spreading resistances as opposed to the vertical resistance of the mirror [7]. Thus, the low lateral resistance of the $n$-doped mirror is effective in decreasing the overall resistance of the $p$-substrate lasers.

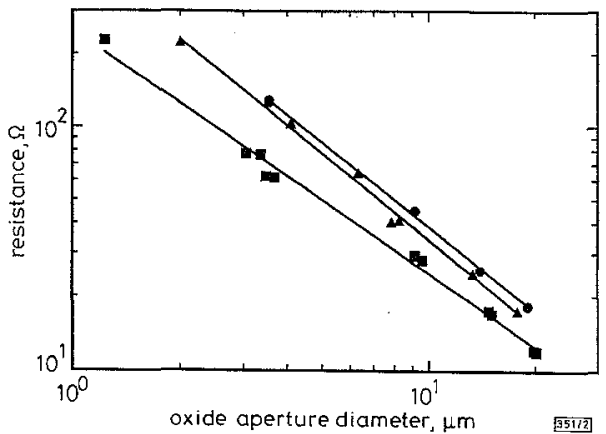

Fig. 2 Resistance against device diameter on logarithmic axes with power law fits for p-substrate and n-substrate VCSELS

p-substrate;

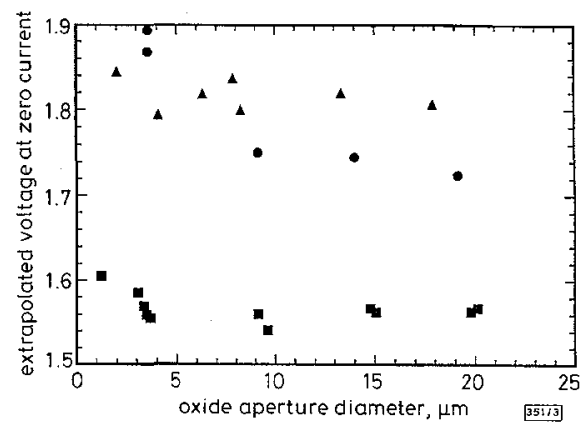

Fig. 3 Extrapolated voltage for zero current against device diameter for p-substrate and n-substrate VCSELs

p-substrate; n-substrate

In addition to a lower differential resistance, the $p$-substrate lasers also have a lower extrapolated voltage at zero current. This voltage is usually dominated by the inversion potential required to achieve lasing at a particular wavelength which is $1.47 \mathrm{~V}$ for $840 \mathrm{~nm}$. Additional contributions arise from non-ohmic voltage drops across heterojunction barriers in the structure. While the doping profile of the mirrors is nominally symmetric within a period, growth kinetics may alter the doping profile and thus change the residual barriers differently for different heterojunctions. Threshold voltages as low as $1.56 \mathrm{~V}$ were measured for the $p$-substrate lasers.

In summary, $p$-substrate VCSELs with characteristics equal to or better than similar $n$-substrate devices have been demonstrated.

Acknowledgment: This work was supported by the United States Department of Energy under Contract DE-AC04-94AL85000 and by the US Air Force Phillips Laboratory. Sandia is a multiprogram laboratory operated by Sandia Corporation, a Lockheed Martin Company, for the United States Department of Energy.

(C) IEE 1997

Electronics Letters Online No: 19970492

10 March 1997

K.L. Lear, H.Q. Hou, J.J. Banas and B.E. Hammons (Sandia National Laboratories, Photonics Research Department, MS 0603/PO Box 5800. Albuquerque, NM 87185-0603, USA)

J. Furioli and M. Osinski (University of New Mexico, Center for High Technology Materials, Albuquerque, NM 87131-6081, USA)

J. Furioli: On leave from Ecole Nationale Supérieure des Télécommunications, Paris, France

M. Osiński: Currently on sabbatical at University of Tokushima, Tokushima, Japan

\section{References}

1 OSINSKI, M., NAKWASKI, W., and VARANGLE, p.: 'Current uniformity and series resistance in vertical-cavity proton-implanted topsurface-emitting lasers grown on $p$-substrates'. (unpublished)

2 HADLEY, M.A., WILSON, G.C., LAU, K.Y., and sMITH, J.S.: 'High singletransverse-mode output from external-cavity surface-emitting laser diodes', Appl. Phys. Lett., 1993, 63, pp. 1607-1609

3 LEI, C., HODGE, L., DUDLEY, J., KEEVER, M., and LIANG, B.: 'High performance OMVPE grown $850 \mathrm{~nm}$ VCSELs on both N-type and P-type substrates'. High-Speed Opto-Electronics for Communications II, Snowbird, Utah, 11-15 August 1996

4 KOPF, R.F., SCHUBERT, E.F., DOWNEY, S.W., and EMERSON, A.B.: 'Nand P-type dopant profiles in distributed Bragg reflector structures and their effect on resistance', Appl. Phys. Lett., 1992, 61, pp. $1820-1822$

5 KIM, S.-I, KIM, Y., KIM, M.-S., KIM, C.K., KIM, S.-K., and LEE, C. 'Carbon doping characteristics of $\mathrm{GaAs}$ and $\mathrm{Al}_{0.3} \mathrm{Ga}_{0.7} \mathrm{As}$ grown by atmospheric pressure metalorganic chemical vapor deposition using $\mathrm{CCl}_{4}{ }^{\prime}, J$. Cryst. Growth, 1994, 141, pp. 324-330

6 CHOQUETTE, K.D., SCHNEIDER, R.P., LEAR, K.L., and GEIB, K.M.: 'Low threshold voltage vertical-cavity lasers fabricated by selective oxidation', Electron. Lett., 1994, 30, pp. 2043-2044

7 LEAR, K.L., and SCHNEIDER, R.P.: 'Uniparabolic mirror grading for vertical cavity surface emitting lasers', Appl. Phys. Lett., 1996, 68 pp. 605-607

8 LEAR, K.L., and CHALMERS, S.A.: 'Overcoming the resistance barrier to better vertical-cavity surface-emitting lasers'. Vertical-Cavity Surface-Emitting Laser Arrays, Los Angeles, 27-28 January 1994

9 PETERS, M.G., THIBEAULT, B.J., YOUNG, D.B., SCOTT, J.W., PETERS, F.H., GOSSARD, A.C., and COLDREN, L.A.: 'Band-gap engineered digital alloy interfaces for lower resistance vertical-cavity surface-emitting lasers', Appl. Phys. Lett., 1993, 63, pp. 3411-3413

\section{Power spectral density of multitrack $(0, G / n$ codes}

\author{
B. Vasić, O. Milenković and S. McLaughlin
}

Indexing terms: Magnetic recording, Codes

A new method for evaluating the power spectral density of multitrack constrained sequences is demonstrated for multitrack $(0, G / l)$ constraints. $(0, G / I)$ constrained sequences are used in magnetic recording systems that employ partial response maximum likelihood (PRML) signalling. Using the proposed approach, the number of states in a directed graph representing the given $(0, G / I)$ constraint is reduced remarkably so that the power spectral density can be easily calculated.

Introduction: In multitrack recording systems [1 - 3], a modulation code translates $N$ unconstrained binary bi-infinite streams $\left\{b_{i}^{(n)}\right\}=$ $\left(\left\{b_{i}^{(n)}\right\}_{n \in Z}\right), 1 \leq i \leq N$ into $N$ channel sequences $\left\{a_{i}^{(n)}\right\}$ that are constrained individually, but satisfy an additional joint (global) constraint. Typical multitrack constraints are generalisations of runlength limited and $(0, G / I)$ constraints $[1,3]$, used in peak detection and partial response (PR) recording systems, respectively [4]. The $I$ constraint is a constraint on the run lengths of consecutive like symbols (phrases) in the sub-sequences $\left\{a_{i}^{(2 n)}\right\}$ and $\left\{a_{i}^{(2 n+1)}\right\}$ of the overall channel sequence $\left\{a_{i}^{(n)}\right\}, 1 \leq i \leq N$. These runlengths should be no greater than $I+I[4]$; this constraint is used to limit the memory of Viterbi detectors used in partial response class IV systems. The $G$ constraint is a constraint on the global sequence $\left\{\mathbf{a}_{i}^{(\mathbf{n})}\right\}$ where the time interval between two transitions in the set of $N$ 'global' streams $\left\{a_{i}^{(n)}\right\}, 1 \leq i \leq N$, is not greater than $G$. The $G$ constraint helps in automatic gain control and clock recovery and is satisfied in a joint manner (since the clock information can be extracted using the transitions in any of $N$ tracks). ' 0 ' in $(0, G / l)$ denotes that minimal phrase lengths are not limited either in the interleaved or in the global streams.

A multitrack $(0, G / I)$ constraint is preferable to a single track $(0$, $G / I$ ) constraint, provided we can read/write on $N$ tracks in parallel In the multitrack case, the $I$ constraint needs to be satisfied on each track, but the $G$ constraint need only be satisfied on one or more tracks. This results in an overall relaxed constraint and a potential increase in the data storage capacity [1]. 